\title{
Acknowledgment to Reviewers of Inventions in 2021
}

\author{
Inventions Editorial Office
}

Citation: Inventions Editorial Office.

Acknowledgment to Reviewers of Inventions in 2021. Inventions 2022, 7,

17. https://doi.org/10.3390/

inventions7010017

Published: 26 January 2022

Publisher's Note: MDPI stays neutral with regard to jurisdictional claims in published maps and institutional affiliations.

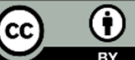

Copyright: $\odot 2022$ by the author. Licensee MDPI, Basel, Switzerland. This article is an open access article distributed under the terms and conditions of the Creative Commons Attribution (CC BY) license (https://creativecommons.org/licenses/by/4.0/).

MDPI AG, St. Alban-Anlage 66, 4052 Basel, Switzerland

Rigorous peer-reviews are the basis of high-quality academic publishing. Thanks to the great efforts of our reviewers, Inventions was able to maintain its standards for the high quality of its published papers. Thanks to the contribution of our reviewers, in 2021, the median time to first decision was 20 days and the median time to publication was 41 days. The editors would like to extend their gratitude and recognition to the following reviewers for their precious time and dedication, regardless of whether the papers they reviewed were finally published:

\begin{tabular}{|c|c|}
\hline Adrian Gligor & Dadmehr Rahbari \\
\hline Adrian Nedelcu & Dagong Jia \\
\hline Ahmed Shaker & Daniela Chicet \\
\hline Ahmet Shala & Danuta Miedzińska \\
\hline Alberto Gianinetti & Dario Pasini \\
\hline Alberto Muscio & David Cabaleiro \\
\hline Aleksei Rozhnov & Den Boychuk \\
\hline Aleksejs Zacepins & Dennis Touliatos \\
\hline Alexandu Vulpe & Diego Antolín \\
\hline Alina Adriana Minea & Dmitriy Shurpik \\
\hline Alon Davidy & Dominika Kaczorowska \\
\hline Amir M. Anvar & Dragos Isvoranu \\
\hline Andrés Feijóo & Dror Malka \\
\hline Andrzej Gajewski & Dwueng-Chwuan Jhwueng \\
\hline Anna Stoppato & Edorta Carrascal Lekunberri \\
\hline Anthony R. Lupo & Eduardo Tolosana \\
\hline Antonio Greco & Elena Popa \\
\hline Antonio Menor-Campos & Eleni Douvi \\
\hline Anuj Kumar & Elliot Taffet \\
\hline Arash Ebneali Samani & Emerson Giovani Carati \\
\hline Arkadiusz Bednarz & Evangelos Bellos \\
\hline Armando Fontalvo & Fabrizio Amisano \\
\hline Aron Perenyi & Fernando Feijoo \\
\hline Arthur E. P. Veldman & Fernando Pérez-Porras \\
\hline Bagh Ali & Florin Postolache \\
\hline Beatriz González & Francisca Rodrigues \\
\hline Bikash Rajan Parida & Francisco Vega Reyes \\
\hline Björn Roscher & François Gannier \\
\hline Buzdugan Mircea Ion & Gabriel Negreanu \\
\hline Capace Alessia & Georgios Ntinas \\
\hline Carl Strathearn & Georgios Pipintakos \\
\hline Chaomin Zhang & Gianvito Scaringi \\
\hline Chin-Ling Chen & Giorgio Lofrese \\
\hline Cristina Campano & Giorgio Sonnino \\
\hline
\end{tabular}

Adrian Gligor

Adrian Nedelcu

Ahmed Shaker

Alberto Muscio

Aleksei Rozhnov

Aleksejs Zacepins

Alexandu Vulpe

Alina Adriana Minea

Andrzej Gajewski

Anna Stoppato

Anthony R. Lupo

Antonio Greco

Antonio Menor-Campos

Anuj Kumar

Arash Ebneali Samani

Arkadiusz Bednarz

Armando Fontalvo

Aron Perenyi

Arthur E. P. Veldman

Bagh Ali

Beatriz González

Bikash Rajan Parida

Björn Roscher

Buzdugan Mircea Ion

Capace Alessia

Carl Strathearn

Cristina Campano
Dadmehr Rahbari

Danuta Miedzińska

Dario Pasini

David Cabaleiro

Den Boychuk

Dennis Touliatos

Diego Antolín

Dmitriy Shurpik

Dominika Kaczorowska

Dragos Isvoranu

Dwueng-Chwuan Jhwueng

orta Carrascal Lekunberri

Elena Popa

Eleni Douvi

Elliot Taffet

Emerson Giovani Carati

Evangelos Bellos

Fabrizio Amisano

Fernando Feijoo

Fernando Pérez-Porras

Florin Postolache

Francisca Rodrigues

Francisco Vega Reyes

Gabriel Negreanu

Georgios Ntinas

Georgios Pipintakos

Giorgio Sonnino 
Grzegorz Wiczyński

Guan Lin

Guillermo Huerta Cuellar

Habib Rahman

Hakeem Niyas

Hanna Kowalska

Henryk Kania

Hesheng Wang

Hongliang Luo

Hongtian Chen

Hyo Jong Lee

Ilaria Campioni

Ilaria Perissi

Iosif-Vasile Nemoianu

Iraklii I. Ebralidze

Irina Cherunova

Ismail Fidan

Jerzy Sowa

Jesus Manuel Fernandez Oro

Jong-Suk Ro

José Gamonales

Jozef Husár

Jui-Yung Chang

Jun-ichi Kadokawa

Katarzyna Lewandowska

Katsuhiko Ariga

Kejun $\mathrm{Wu}$

Khairullo Faizullaevich Makhmudov

Kimmo Kauhaniemi

Klaus Bogenberger

Kok Hoe Wong

Kotb Tawfiq

Kung-Ming Chung

Kwok-wing Chau

L'udmila Dulebová

Laibao Zhang

László Almásy

Laura Bulgariu

Lecturer Carmen Rodica Pop

Leila Marek-Crnjac

Lucian Dulău

Lucjan Kozielski

Luis Norberto López De Lacalle

M. Estela Peralta

Maciej Dzikuć

Magdalena Skarzynska

Magdalena Żuk

Mahmoud A. Khader

Manuel Jesús Espinosa

Marcello Trovati

Marco Fontanelli
Marcos Tostado-Véliz

María De Las Nieves González

Mário W. L. Moreira

Mariusz Ciesielski

Matthew Hobbs

Mauro Chinappi

Md Rabiul Islam

Md Saiful Bari

Michal Borecki

Michał Strzelecki

Mihai Zaharia

Mingyang Lu

Mircea Viorel Drăgoi

Miroslaw Bajda

Mohammad Safeea

Mohammad Shojafar

Mohammad Yaghoub Abdollahzadeh Jamalabadi

Mohammadali Hedayat

Mohsen Saffari Pour

Moussa Boukhnifer

Muhammad Abdul Qyyum

Nabilah Shamim

Nataliya Sakharova

Nathalie Sauer

Nicolae Tudoroiu

Nikolaos V. Kantartzis

Nikolay Ivanov

Nikos Karayiannis

Noura Dosoky

Nuria Huete

Octavian Fratu

Oday Ibraheem Abdullah

Olga Orynycz

Oscar Alfranca

Paolo Mercorelli

Paraskevas Nikolaou

Parham Nooralishahi

Paweł Krzaczek

Peter Ampim

Peter Bindzár

Poushali Das

Prodip K. Das

Rafael Sola-Guirado

Randy Oliver

Raouf Senhadji-Navarro

Reiner Kriesten

Ricardo Vardasca

Rizwan Ali Naqvi

Robert Thresher

Rodica Margaoan

Rodolfo Picchio 
Rosen Mitrev

Rui Antunes

Rui M. S. Cruz

Ryo Kohsaka

Salim Lamine

Salman Arain

Sandra Costanzo

Silvana Alfei

Soheil Jafari
Susmit Bagchi

Syed Basit Ali Bukhari

Tatiana Morosuk

Tomasz Kałuża

Younes El Kacimi

Yudhi Adhitya

Yun Zhang

Zbigniew Leonowicz 